\title{
Mass Spectrometry Imaging of Microbial Interactions using Matrix-Enhanced Laser Desorption/Ionization Fourier-Transform Ion Cyclotron Resonance Mass Spectrometry
}

Christopher R. Anderton, ${ }^{1}$ Rosalie K. Chu, ${ }^{1}$ Jared B. Shaw, ${ }^{1}$ Erika M. Zink, ${ }^{1}$ Carolyn A. Zeiner, ${ }^{2}$ Dennis Trede, ${ }^{3}$ Colleen M. Hansel, ${ }^{4}$ and Ljiljana Paša-Tolić ${ }^{1}$

${ }^{1}$ Pacific Northwest National Laboratory, Richland, WA 99354

${ }^{2}$ School of Engineering and Applied Sciences, Harvard University, Cambridge, MA, USA

${ }^{3}$ SCiLS GmbH, Bremen, Germany

${ }^{4}$ Marine Chemistry and Geochemistry Department, Woods Hole Oceanographic Institution, Woods Hole, MA, USA

Mass spectrometry imaging (MSI) methods offer the ability to gain both chemical and spatial information from biological samples of interest. In particular, matrix-enhanced laser desorption/ionization (MALDI) MSI facilitates detection of a variety of intact biomolecular species (e.g., lipids, peptides, and proteins) with spatial resolution regularly $<20 \mu \mathrm{m}$ [1]. When analyzing complex systems, like biological samples, the need for high mass spectral resolution and high mass accuracy measurements to correctly identify and map molecules of interest is warranted. Fourier transform ion cyclotron resonance (FTICR) MS offers the highest mass resolving power and mass accuracy of any mass analyzer and has demonstrated greatly enhanced MSI performance [2]. Here, we will demonstrate the advancements our lab has made in MALDI MSI. In particular, we focus on characterizing the interactions of Eumycete fungi, key organisms in carbon degradation within the environment. We also explore new sample preparation methods and data analysis workflows.

The advantages of using high performance mass spectrometers for studying biological material is illustrated in Fig. 1A, where many more spectral features can be resolved using FTICR verses more traditional MS (ToF) [2]. However, to attain unequivocal identification of detected molecules, tandem mass spectrometry must be used in conjunction with high performance mass spectrometers. We have equipped our high magnetic field (15T) FTICR-MALDI MS (SolariX, Bruker) with a UV laser (193 nm, ArF excimer, Coherent ExciStar XS) for UV photodissocation of selected ions within our ICR analyzer cell. Fig. 1B demonstrates the tandem MS capabilities of this method, where we observe a more comprehensive fragmentation of a lactosylceramide (LacCer) molecule than conventional tandem MS approaches.

Sample preparation of agar-based microbial samples has been a significant limitation to MSI of microbial communities with MALDI, with only minor improvements being reported to the method the Dorrestein Research Group developed [3]. This basic method entails dry application of the matrix onto the hydrated agar sample by using a sieve, followed by agar dehydration to form a crystalline surface ready for MALDI analysis. We have explored a series of other agar conditions, sample adhesion, and matrix applications routes to advance the ability of MALDI MSI for imaging microbial communities inculcated on agar. In this we have also investigated the use of a robotic sprayer (TM-Sprayer, HTX Imaging) for matrix application in attempt to make highly homogenous, reproducible matrix films, while maximizing analyte extraction into the matrix crystal.

Finally, the complexity and size of high mass resolution MSI data presents challenges in data 
processing. Opening and caching MSI files that are typically tens to hundreds of gigabytes in size, generating peak list with sufficiently tight tolerances of hundreds of peaks, and elucidating biological signatures of interest can be extremely challenging. We have been utilizing an advanced MSI processing software (SCiLS Lab), previously developed for ToF MSI workflows, for spatial characterization of detected molecules from microbial samples [4,5].

References:

[1] B Spengler, Anal. Chem. 87 (2015), 64.

[2] D F Smith, A Kiss, F R Leach III, E W Robinson, L Paša-Tolić, and R M A Heeren, Anal. Bioanal. Chem. 405 (2013), 6069.

[3] J Y Yang, V V Phelan, R Simkovsky, J D Watrous, R M Trial, T C Fleming, R Wenter, B S Moore, S S Golden, K Pogliano, and P C Dorrestein, J. Bacteriol. 194 (2012), 6023.

[4] T Alexandrov, M Becker, S-O Deininger, G Ernst, L Wehder, M Grasmair, F von Eggeling, $\mathrm{H}$ Thiele, and P Maass, J. Proteome Res. 9 (2010), 6535.

[5] This work was performed at EMSL, a national scientific user facility sponsored by the Office of Biological and Environmental Research, U.S. Department of Energy (DOE). EMSL is located at PNNL, a multidisciplinary national laboratory operated by Battelle for the U.S. DOE. We would like to thank Professor Pieter Dorrestein (UCSD) for his many useful discussions, and Mr. Alain Creissen is thanked for his contributions and discussions in optimizing the TM-Sprayer for matrix applications.
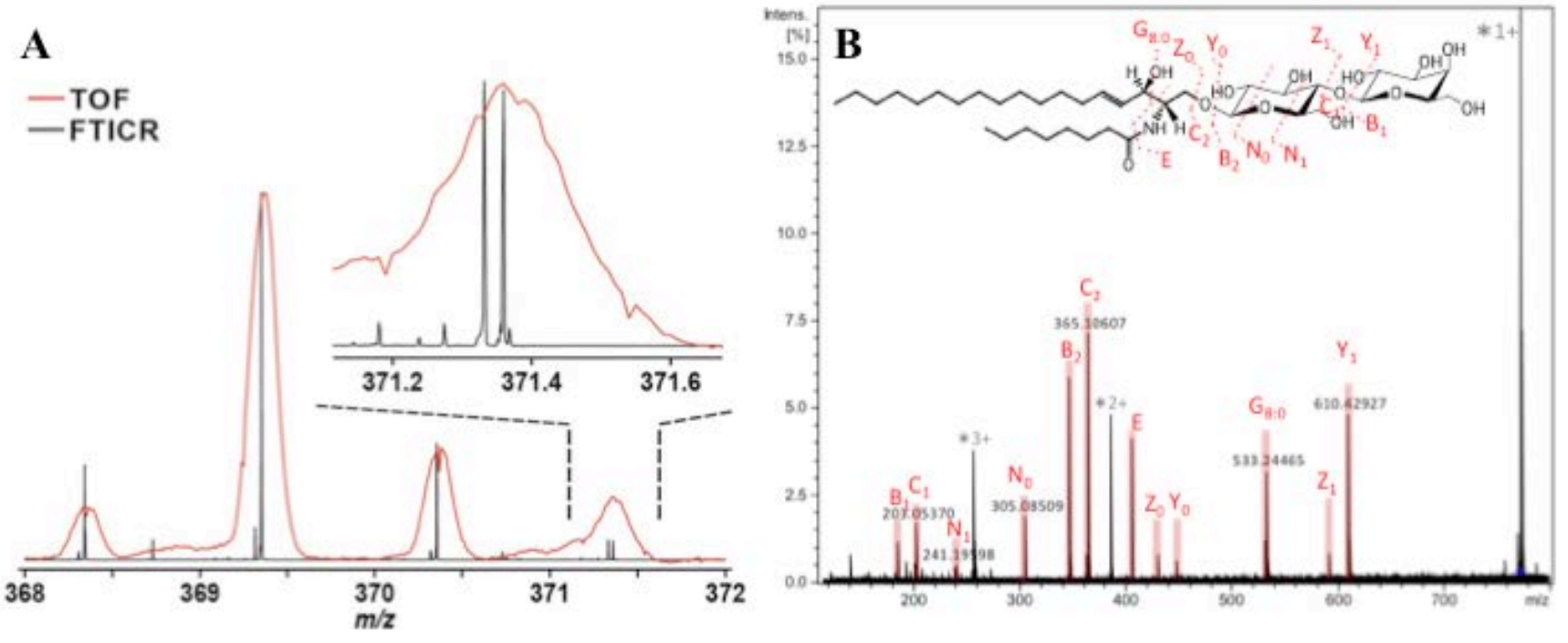

Figure 1. (A) Spectral overlay of ToF and FTICR MS data taken from the same rat brain section. This figure was modified from reference [2]. (B) MALDI-UVPD mass spectrum of the sodium adduct of LacCer (d18:1/8:0). 